

\section{Endometriosis presenting as an obstructed femoral hernia: a case report}

*R MAKUNIKE, *C MURONDA, **SD SABURI

\section{Introduction}

Endometriosis has been reported in many different pelvic, and extra-pelvic sites. We have not previously seen a report of endometriosis in a femoral hernial sac. We report one such case.

Cent Afr J Med 2001;47(7):184-6

"Histopathology Department

University of Zimbabwe Medical School

*:Medical Centre

37 Baines Avenue

Harare
Correspondence and reprints to:

Dr RT Makunike

Histopathology Department

University of Zimbabwe Medical School

PO Box A178, Avondale, Harare

Zimbabwe

Fax 26304703857

Histopath@med-sch.uz.zw 


\section{Case Report}

A 43 year old black female presented to a general surgeon with a three month history of swelling in the right groin. The swelling was initially episodic, although she had not specifically noticed a relationship with the menstrual period. On further questioning, she admitted that the episodes may have beèn approximately three to four weeks apart. The mass was initially fluctuant and reducible, but at the time of presentation, the mass was fixed and the pain persistent.

On examination, there was a firm globular tender swelling in the right groin below the inguinal ligament. The overlying skin was discoloured and the mass was not reducible.

The femoral hernia was explored and repaired using McEvedy's approach. The hernial sac did not contain omentum as expected, but contained haemorrhagic tissue. The tissue was submitted for histology. The patient's immediate post operative period was uneventful. She was referred to a gynaecologist for follow up. Abdominal ultra-sound scan and laparascopy did not show any evidence of endometriosis. She remains symptom-free, 13 months after the operation.

\section{Pathology Findings.}

A $30 \times 20 \times 15 \mathrm{~mm}$ fatty mass with areas of haemorrhage was received. The tissue was processed in a Shandon processor overnight and embedded in paraffin blocks. Multiple sections were cut at 5um levels and stained with Haematoxylin and Eosin. Microscopic examination showed endometrial glands embedded in fibro-adipose tissue in which were prominent vascular channels(Figures I and II). The glands were accompanied by stroma with abundant haemosiderin laden macrophages and focal decidualisation typical of endometriosis. Other areas showed cystic spaces devoid of an epithelial lining and containing necrotic debris with haemorrhage.

\section{Discussion}

Endometriosis is defined as the presence of endometrial glands outside the uterus and its histological diagnosis is dependant on finding endometrial glands, endometrial stroma and haemorrhage in a site outside the uterus. At least two of these features must be present in order to diagnose endometriosis. ' Our case was readily diagnosed as all three elements were present. In more advanced lesions, however, the diagnosis may be difficult and elude the uninitiated or unsuspecting pathologist, if the glands are not readily seen.

The presence of the spindle cells seen in the stroma, together with frank haemorrhage or haemosiderin laden macrophages, should alert the pathologist to this possibility, and further sampling should be carried out. In addition, when the lesion is advanced, only necrotic pseudoxanthomatous nodules are seen and these must be distinguished from infectious granulomas.
Figure 1: Medium power view showing variably sized endometrial glands with stroma in a fibro-adipose tissue.

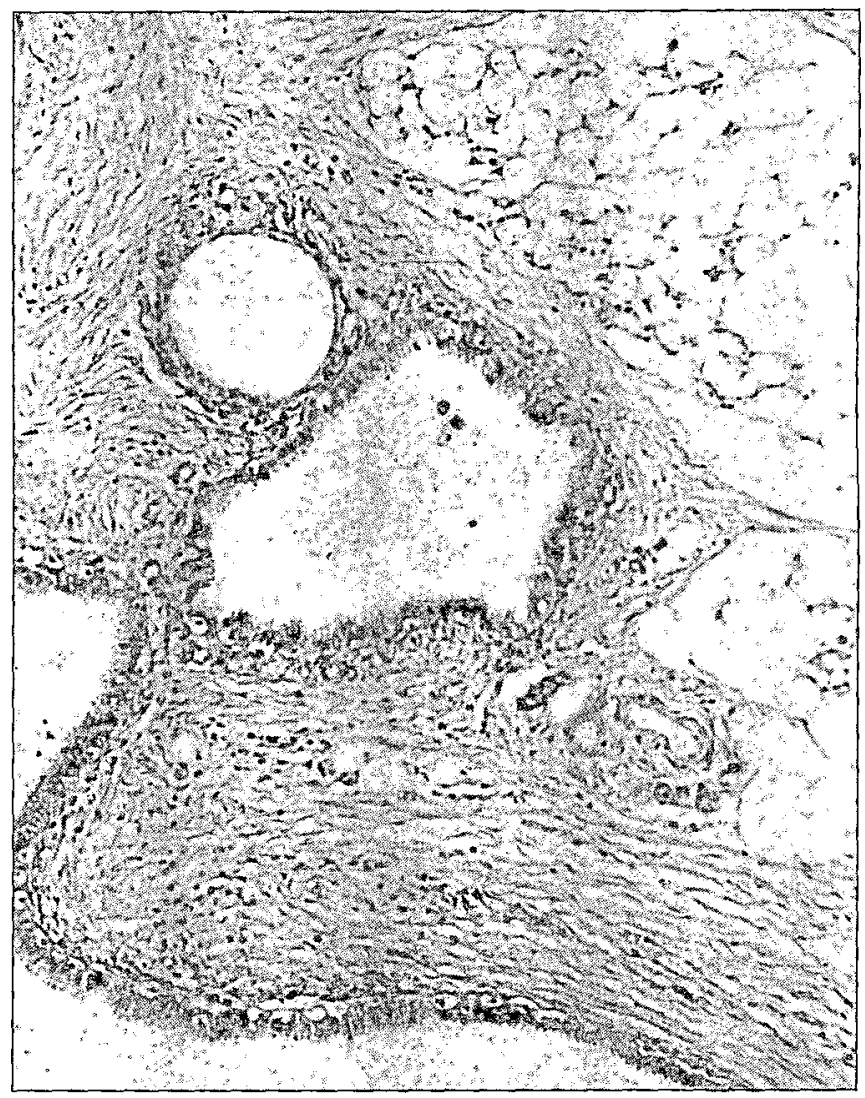

Figure 11: High power view showing gland lined by columnar epithelium.

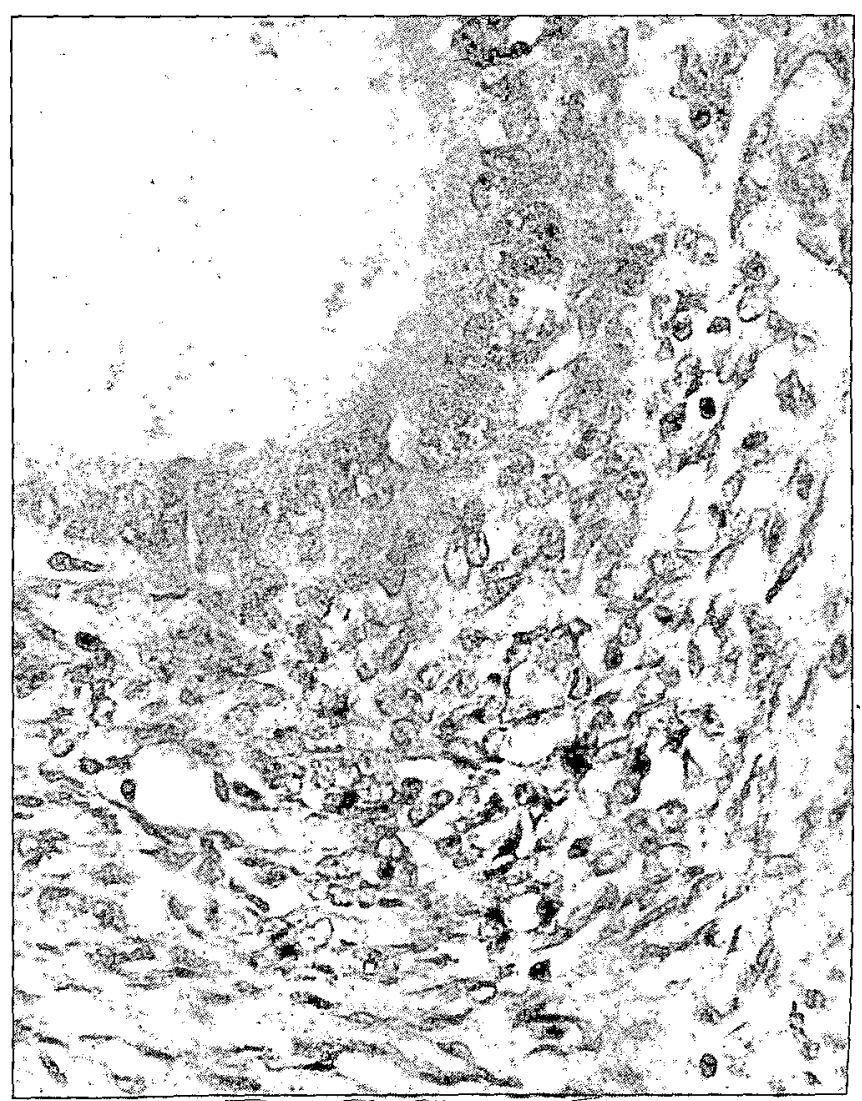


Endometriosis has been described in numerous sites including the $\backslash$ cervix, vagina, vulva, pouch of Douglas, ovary, fallopian tube, uterine ligaments, appendix, small and large bowel, bladder, ureters, pelvic peritoneum, hernial sacs, lymph nodes, kidneys, skin, skeletal muscle, peripheral nerves, pleura, lung and nasal cavity. ${ }^{2-6}$ We know of only one other case reported in the femoral region. ${ }^{7}$ Recalde and Majmudar reported a case of a 27 year old white woman, para 1 , gravida 1 who presented with a persistent buldge in her groin.

There has been much speculation on the pathogenesis of endometriosis. Sampson proposed a Retrograde Menstruation "Regurgitation Theory". This theory presupposes that some or all women, to some extent experience some degree of retrograde menstruation into the fallopian tube and ovary. ${ }^{8}$ This theory accounts for endometriosis occurring in the adnexea but not in distant sites. The metaplastic theory supports origin from metaplasia of coelomic derived epithelium. ${ }^{9}$ This theory explains endometriosis in other organs i.e. bowel, but again, not in distant sites. The metastatic theory states that endometrial glands are transported via the vascular or lymphatic routes. ${ }^{10}$ This theory is borne out by the occurrence of endometriosis in distant sites i.e. the nasal cavity.' The presence of endometriosis in the femoral canal could possibly be explained by the metaplasia theory if the lesion was present in the bowel, associated with both an inguinal and femoral hernia. Alternatively, it can be explained by the Metastatic theory.

Endometriosis was thought to be more common in Caucasians but other studies show a similar frequency of the disease in Asians and Africans. ${ }^{11,12}$, This case highlights the possibility of this lesion occurring in black women and in addition its occurrence at an unusual site. Some authors have suggested that all women have "retrograde menstruation" to some degree. ${ }^{13}$ Therefore, the diagnosis of endometriosis should be excluded in any woman with a history of cyclical pain. Establishment of the correct diagnosis will enable the patient to receive the appropriate therapy and reduce the morbidity of what is often a debilitating disease.

\section{References}

1. Czernobilsky B. Pathology of the female genital tract. Endometriosis. Vol.5. 1043-60.

2. Majeski J. Scar endometriosis manifested as a recurrent inguinal hernia. South Med $J$ 2001;94(2):247-9.

3. Miskry T, Rauch M, Magos A, Farhat S. Incisional hernia involving a fallopian tube and mimicking scar endometriosis. Obstet Gynecol 2000;95 (6 Pt 2):1028.

4. Nirula R, Greaney GC. Incisional endometriosis: an under-appreciated diagnosis in general surgery. $J$ Am Coll Surg 2000;190(4):404-7.

5. Quagliarello J, Coppa G, Bigelow B. Isolated endometriosis in an inguinal hernia, Am J Obstet Gynecol 1985;152:688-9.

6. Wolf Y, Haddad R, Werbin N, Skornick Y, Kaplan O. Endometriosis in abdominal scars:a diagnostic pitfall. Am Surg 1996;62(12):1042-4.

7. Recalde AL, Majmudar B.Endometriosis involving the femoral vein. South Med J. 1977:70(1):69,74.

8. Sampson JA.The development of the implantation theory for the origin of peritoneal endometriosis. $\mathrm{Am}$ J Ostet Gynecol 1940;40:549-57.

9. El-Mahgoub S, Yassen S. A positive proof for the theory of coelemic metaplasia. Am J Obstet Gynecol 1980;137:137-40.

10. Sampson JA. Metastatic or embolic endometriosis, due to the menstrual dissemination of endometrial tissue into the venous circulation. Am J Pathol 1927;3:93-109.

11. Houston DE, Noller KL, Melton J III, Selwyn BJ. The epidemiology of pelvic endometriosis. Clin Obstet Gynecol 1988;31:787-800.

12. Chatman DL. Endometriosis and the black woman. $J$ Reprod Med 1976;16:303-6.

Halme J, Hammond MG, Hulka JF. Retrograde menstruation in healthy women and in patients with endometriosis. Obstet Gynecol 64:151-4. 


\section{(c) (1) (9)}

This work is licensed under a

Creative Commons

Attribution - NonCommercial - NoDerivs 3.0 License.

To view a copy of the license please see:

http://creativecommons.ora/licenses/bv-nc-nd/3.0/ 